\title{
Consideration of uncertainty of information on load and generation values under daily reconfiguration of primary distribution network
}

\author{
Irina Golub ${ }^{1, *}$, OlegVoitov$^{1}$, Evgeny Boloev $^{1}$ and Lyudmila Semenova ${ }^{1}$ \\ ${ }^{1}$ Melentiev Energy Systems Institute, 664033 Lermontov str., 130, Irkutsk, Russia
}

\begin{abstract}
Intelligent electric networks make it possible to remotely control switchings, thus preventing overloads and enhancing reliability of power supply to consumers. In the paper, the daily reconfiguration of a primary distribution network is considered as a means to reduce energy losses in the network. An algorithm applied for the reconfiguration is based on the methods of the theory of graphs and includes a high-speed program for load flow calculation. The research is focused on the impact of renewable generation and active demand with loads changing depending on daily variation in electricity price on the reduction in losses at reconfiguration. An algorithm is proposed to optimize load curves of load-controlled consumers. The calculation of probabilistic load flows is applied to assess the impact of the uncertainty of nodal power forecast on energy loss reduction at reconfiguration. The results of the research demonstrate the effectiveness of the proposed approaches, and are illustrated by a 33-node test distribution network.
\end{abstract}

\section{Introduction}

Most of the energy losses in the electric power systems are known to occur in distribution networks. These losses make up 10-13\% of all the generated electric energy. The problem of energy loss reduction in the distribution network that consists of a medium voltage distribution network (primary) and low voltage distribution network (secondary) remains important for engineers and researchers. This paper is concerned with an issue of reducing energy losses in the primary distribution network which feeds secondary distribution network transmitting energy to end consumers.

The primary distribution networks are weakly closed, however owing to normally open tie switches between feeders, they operate as open. Apart from the normally open tie switches, there are normally closed sectionalizing switches that can disconnect one of the feeder sections. Closing of a tie switch and opening of a respective closed switch allow us to obtain a new radial configuration of the distribution network. Such a procedure called reconfiguration algorithm enables us not only to enhance power supply reliability but also to reduce energy losses and hence energy consumed from supply network, currents in tie lines, voltage losses in distribution network, and, moreover, more fully involve renewable generation in the case it is used in the network.

In a traditional passive distribution network power flows go from primary distribution substation being the only supply source along the branches of a tree scheme to a leaf node. Voltage deviations increase as the distance between source and the dangling node rises.
Thus, voltage behavior in the traditional distribution network is predictable and its monitoring is unnecessary.

Transition to active distribution network is related to the use of distributed generation, energy storage systems, and active demand. Directions of flows in the branches of the active distribution network vary during a day. The load nodes can become generator nodes and voltage deviations can exceed admissible values. Therefore, such networks must be monitored.

In passive distribution network the flows and voltage are measured only at the primary distribution substation. The conditions of the secondary distribution substation are normally unknown [1]. In the future intelligent distribution networks active distribution networks will be part of advance metering infrastructure [2] and operator of distribution network will receive data on loads and generation in real time. In this paper, however, we assume that the distribution network operator has a short-term forecast of active and reactive loads and active power generation of renewable energy sources represented by wind turbines and photovoltaics. Daily load curves and generation schedules of renewable energy sources are taken from the research [3] which is also devoted to the problem of energy loss reduction under hourly reconfiguration of distribution network. Energy losses per hour are numerically equal to average power losses during an hour. Therefore, in an analysis of losses for a concrete hour we will consider power or energy losses.

Since the forecasts of loads and generations contain errors, the most pressing problems are the assessment of an impact of forecast errors on the errors of energy losses in the distribution network under its daily

\footnotetext{
* Corresponding author: golub@isem.irk.ru
} 
reconfiguration, and the assessment of reconfiguration justifiability under uncertain initial information.

In our research reconfiguration is considered as the main tool of reducing power losses in the distribution network. A great many algorithms for solving this problem are presented in publications. According to [4] they include the algorithms of mixed integer and nonlinear programming, and heuristic methods such as Genetic Algorithms, Artificial Neural Networks, Ant Colony, Harmony Search, and Tabu Search. The other algorithms involve linear load flow to calculate losses at network reconfiguration, because nonlinear load flow is considered to be time-consuming. In [4], the authors solving the problem of distribution network reconfiguration apply both the algorithms for the construction of a maximum spanning tree and the simplified approaches to specification of currents and power losses. It is probably this simplification that did not allow the researchers to find better solutions which were obtained with the help of other algorithms, for example, in [5].

The proposed reconfiguration algorithm is based on the methods known in the theory of graphs and intended for the construction of a maximum spanning tree [6] and determination of branches of independent loops by their chords [7]. These methods are included in the high-speed program of steady state calculation, which is the main advantage of the proposed algorithm compared to the reconfiguration algorithm in [4]. The main stages of the algorithm that does not take into consideration the presence of several generation sources in the distribution network are presented in Section 1.

Section 2 is focused on the study of the impact of renewable generation and/or reconfiguration on losses. The impact of renewable generation on the number of switchings at reconfiguration is studied, and the possibility of determining a scheme of switchings providing the minimum daily losses within the entire range of variations in nodal power is demonstrated. The assessment of losses was illustrated along with the expected characteristic of voltage, whose deviations decline with reduction in losses. Moreover, the impact of renewable generation and reconfiguration on the currents in distribution network is analyzed.

Section 3 is concerned with another possibility of reducing losses of energy and currents in tie lines. This is regulation of hourly energy consumption by load controlled consumers, which leads to a change in the daily load curve. An overview of the methods for active demand control is presented in [8]. The authors of [8] solve this problem by the linear programming method.

In Section 4 the linear analytical method of probabilistic load flow is applied to assess the impact of uncertainty of the day-ahead forecast of loads and generation on energy losses caused by the distribution network reconfiguration. In [9], the impact of uncertainty of the initial information on losses due to reconfiguration is studied by using the probabilistic load flow based on the point method.

\section{A reconfiguration algorithm}

Power losses in a closed network are normally lower than in an open network [10]. This condition is not met in the event of circulating and interchange currents in the loop. The circulating currents are caused by phaseshifting devices in the loop and the interchange currents are caused by several supply sources. Nevertheless, in the case of reconfiguration, it is necessary to make where possible power losses in the open network close to the losses in the closed network.

This condition can be met if we construct a spanning tree with the minimum sum of power losses in its chords in the closed network. Such a criterion however may prove unacceptable, if resistance in the branch with high current virtually equals zero. Therefore, the criterion of the minimum sum of absolute values of currents in chords is more reliable. The maximum spanning tree with the minimum currents in chords can be constructed using the known method of the theory of graphs [6]. In this method, the network graph branches are arranged in a descending order of absolute values of currents in them. In a cycle, depending on the number of branches, the branches, in which either one or both nodes are not yet included in the tree, are successively connected to the spanning tree. In the event that both nodes of the branch enter the tree, such a branch is called a chord. The algorithm divides all the branches of the network graph into the branches of the spanning tree and the chords.

To determine branches of each independent loop by its chords, we construct a submatrix of a block of trees of the second incidence matrix [7]

$$
N_{t}=-M_{c h}^{T}\left(M_{t}^{T}\right)^{-1} .
$$

This matrix contains the number of rows equal to the number of chords and the number of columns equal to the number of tree branches, where $M_{t}$ and $M_{c h}$ - are submatrices of the first incidence matrix, that correspond to the branches of the tree and chords. Expression (1) serves as a basis for the topological algorithms [10] used to construct the first incidence matrix $\left(M_{t}^{T}\right)^{-1}$ inverse to the block of trees and the second incidence matrix $N_{t}$

The algorithm consists of two steps. In the first step, we determine the composition of disconnected chords. In the beginning, all the switches are closed. Then, the load flow is calculated in the number of iterations equal to the number of independent loops in the graph of the distribution network and a chord with the minimum current to be open is determined.

In the second step, we check the possibility of reducing power losses by replacing the chords opened in the first step by the branches of the spanning tree in a loop connected to the chord. For each of the chords considered in the first step we do the following: close the next chord; identify the branches of the loop connected to it; calculate the load flow; mark the nodes to the left and to the right of the chord nodes that have the degree above two; simulate a successive disconnection of branches situated between such nodes, with calculation of load flow and determination of total losses; open the 
previous or new chord that corresponds to the minimum total losses. If the loop does not have nodes with the degree above 2 , then the disconnection of all branches of the loop is simulated. It is recommended to repeat the second step of the algorithm once again.

\section{Hourly reconfiguration of distribution network}

Power losses under optimal configuration, which were taken for some conditions, will not be optimal in the entire range of variation in the nodal powers. This means that reconfiguration should follow changes in loads and generation. We will illustrate the efficiency of applying the proposed algorithm of distribution network reconfiguration to reduce losses at each hour of a daily load curve and generation.

The algorithm performance and subsequent analysis of factors that have an effect on the losses are illustrated by a 33-node scheme [11] (Fig. 1), including 37 sectionalizing switches and 5 tie switches. Daily curve of an hourly variation in active, Fig. 2 a, and reactive power loads at nodes 1-33 and variations in active power generated by renewable energy sources at nodes $6,9,13$, 32 , Fig. 2b, are taken from [3]

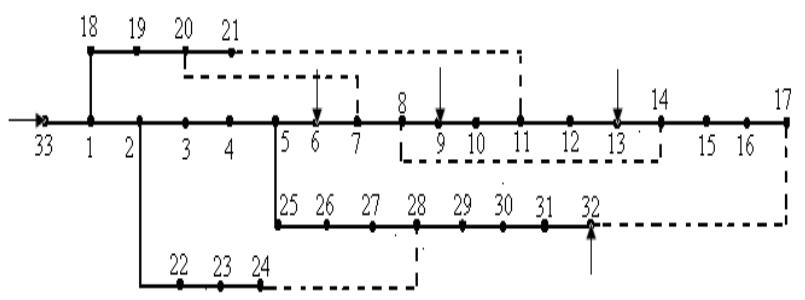

Fig. 1. Scheme of a 33-node distribution network. The lines with the tie switches are shown by dotted lines.
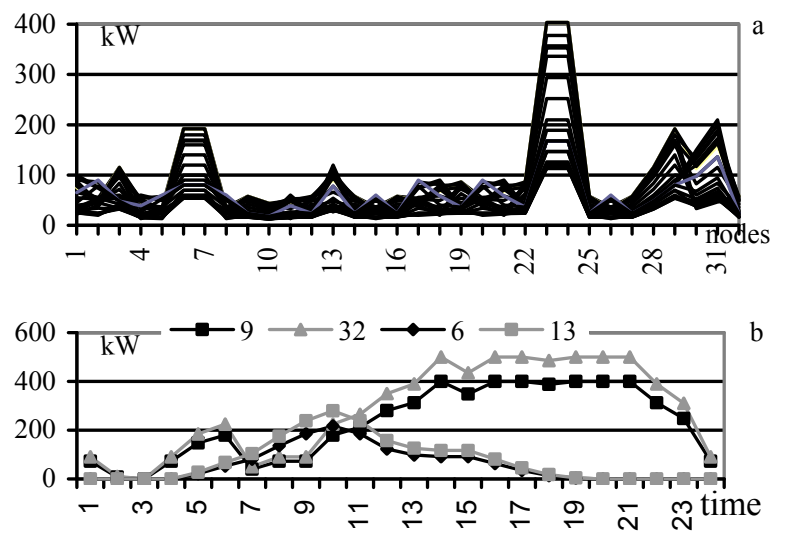

Fig. 2. Variation in active power of loads at the nodes of distribution network, (a) and active power of renewable energy sources (b) during a day.

Figures 3 a, b demonstrate the performance of the reconfiguration algorithm for a test network for loads and generation at hour 11. A reduction in the losses for the option with renewable generation after opening three tie lines on the first step is caused by the interchange currents.

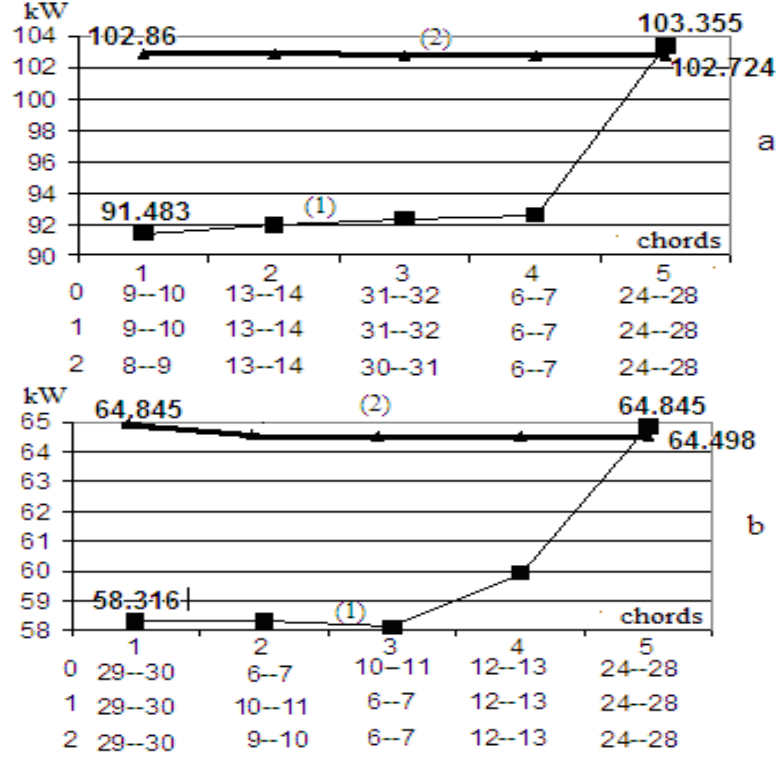

Fig. 3. Change in power losses at reconfiguration of a test network, without (a) and with (b) renewable generation, 0 - an initial composition of chords, arranged in a descending order of currents in them; 1 - chords obtained after the first step (1); 2 chords obtained after the second step (2).

Figure 4 presents the values of daily energy losses and curves of variation in hourly energy losses in a distribution network with and without reconfiguration and renewable energy sources.
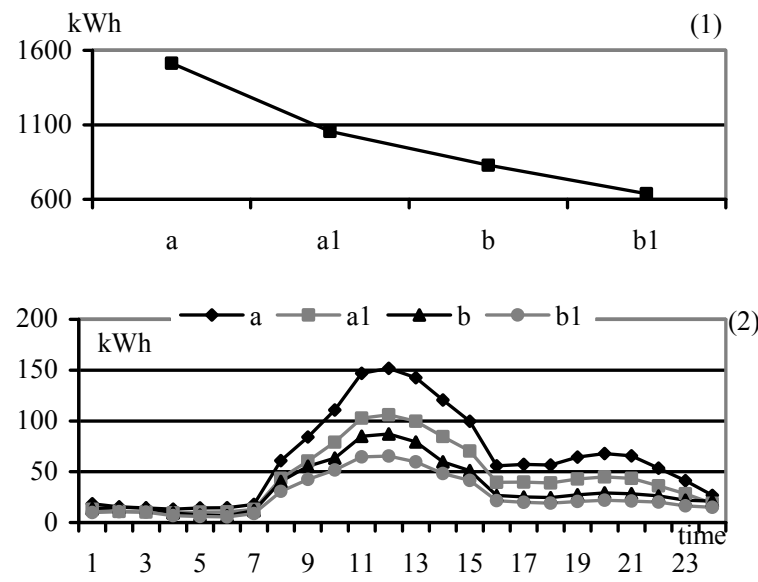

Fig. 4. A change in the daily (1) and hourly (2) energy losses in distribution network with and without reconfiguration and presence of renewable energy sources, a - without renewable generation and without reconfiguration, a1 - without renewable generation and with reconfiguration, $b$-with renewable generation and without reconfiguration, b1 - with renewable generation and with reconfiguration.

Comparison of the losses made it possible to draw the following conclusions:

- Renewable generation has a greater effect on losses than network reconfiguration. The daily energy losses make up $829.37 \mathrm{kWh}$ and $1055.95 \mathrm{kWh}$, respectively;

- $\quad$ Simultaneous use of renewable generation and reconfiguration provides more than a two-fold reduction in losses equal to $636.98 \mathrm{kWh}$ compared to the losses in the distribution network without renewable generation and reconfiguration, $1513.97 \mathrm{kWh}$; 
- $\quad$ The involvement of renewable energy generation leads not only to a change in power flows in lines, but also to a change in their directions, and as a result, to an increase in the total number of switchings at daily reconfiguration. Their number without renewable energy sources is 16 and with renewable energy sources -76 .

A great number of switchings can lead to a switching cost commensurate with or exceeding the cost of reducing energy losses at reconfiguration. Figure 5 illustrates the possibility of selecting a constant distribution network configuration ensuring the minimum daily energy losses.

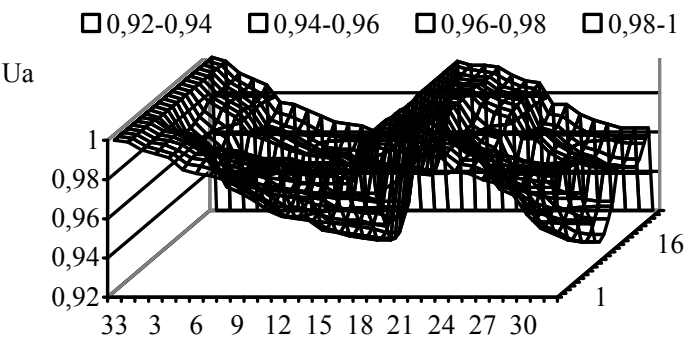

$\square 0,92-0,94 \quad \square 0,94-0,96 \quad \square 0,96-0,98 \quad \square 0,98-1$
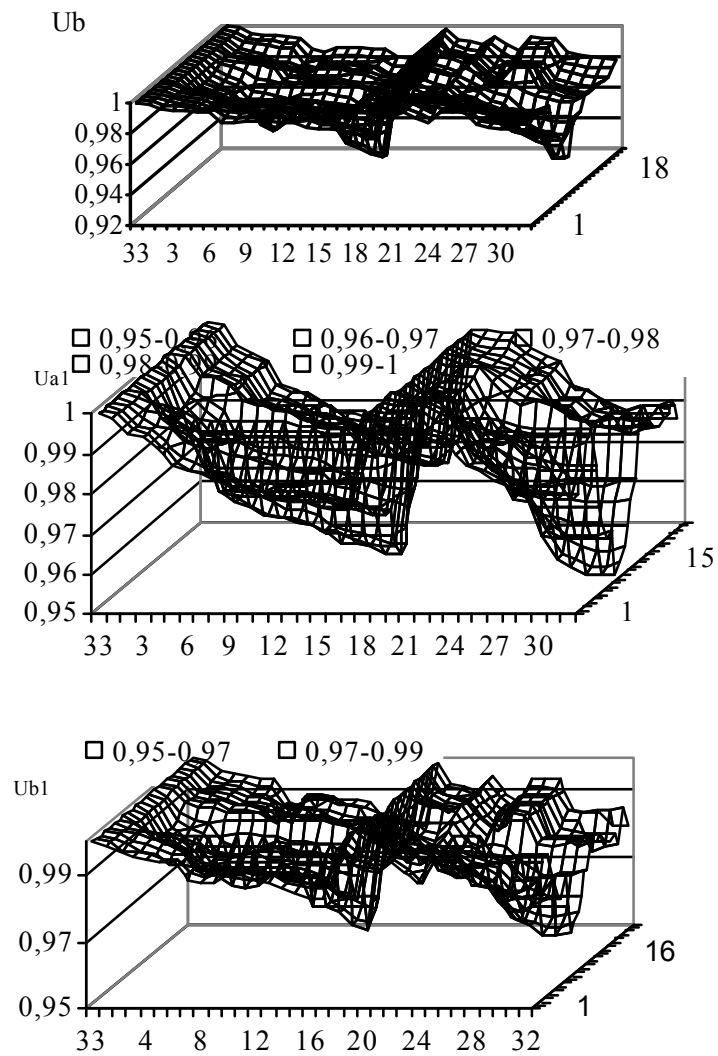

Fig. 6. Hourly variations in voltage at nodes and current in branches of the test network with and without reconfiguration and renewable generation, Ua, Ia - (voltage and current ) without renewable generation and reconfiguration, $\mathrm{Ub}$, Ib - without renewable generation and with reconfiguration, Ua1, Ia1 - with renewable generation and without reconfiguration, Ub1, Ib1- with renewable generation and with reconfiguration.

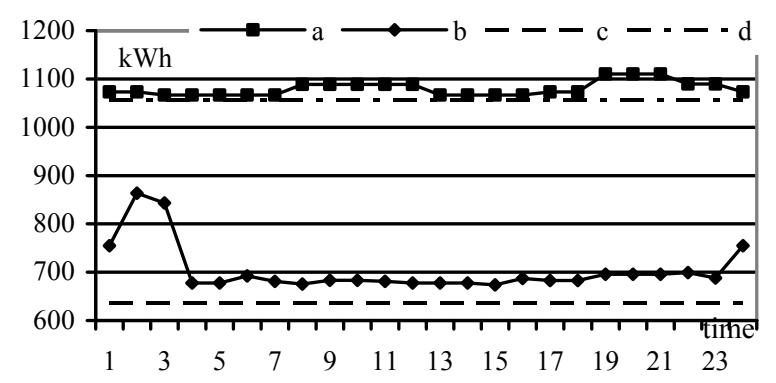

Fig. 5. Comparison of daily energy losses at constant configuration of distribution network without renewable generation $-a$, and with renewable generation $-b$, with the daily losses obtained for the optimal reconfiguration without renewable generation $-\mathrm{c}$ and with renewable generation $-\mathrm{d}$.

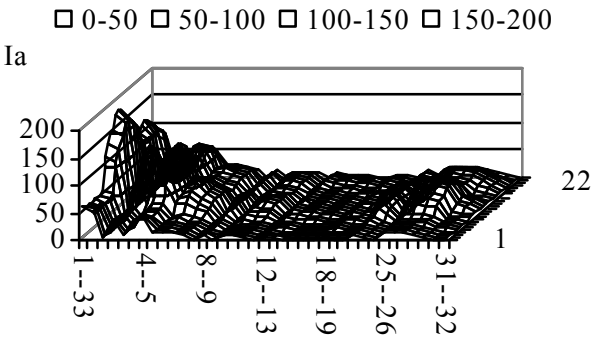

$\mathrm{Ib}$
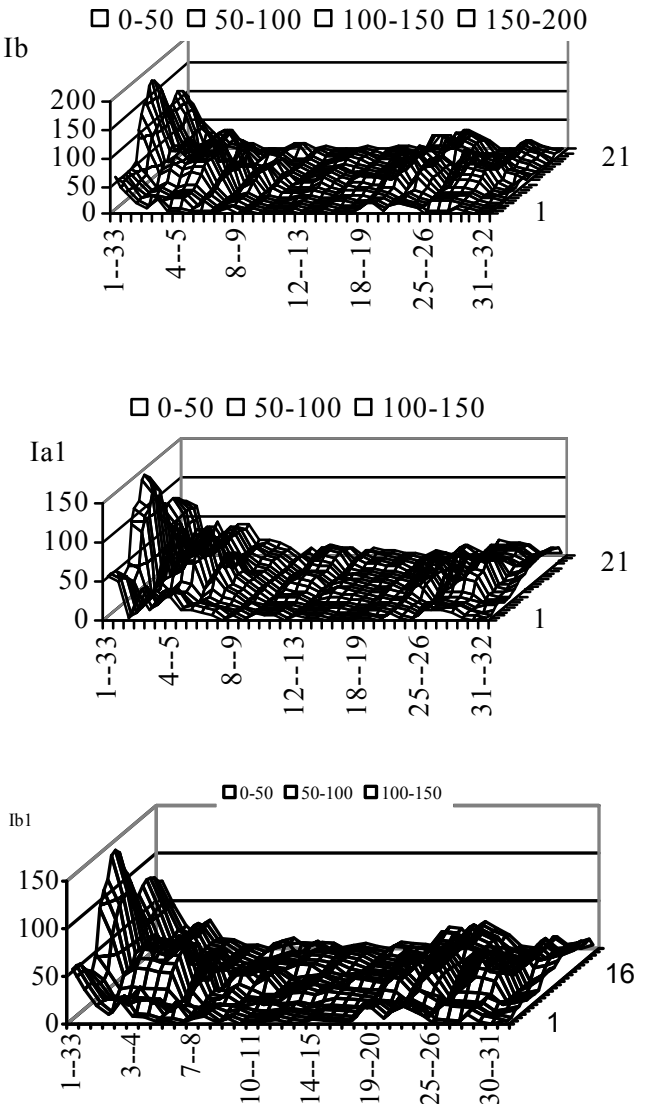
Figure 5 shows the values of daily losses under constant distribution network configuration coinciding with each of the hourly configurations obtained for it with and without renewable energy generation in the network. In the former case such losses are compared with daily energy losses in the network without renewable generation but with reconfiguration, that are equal to $1055.95 \mathrm{kWh}$, and in the latter case, they are compared with the losses in the network with renewable generation and with reconfiguration, that are equal to $636.9 \mathrm{kWh}$.

Comparison of daily losses shows that under constant configuration of distribution network with renewable generation that corresponds to hour 15, losses $(673.6 \mathrm{kWh})$ will be higher than the optimal losses $(636.9 \mathrm{kWh})$ by $36.7 \mathrm{kWh}$. Thus, a loss reduction by $36.7 \mathrm{kWh}$ required 76 switchings. In the case study without renewable generation the minimum losses under constant daily configuration coinciding for hours 3-7 and 13-16 differ from the losses under optimal configuration only by $10.5 \mathrm{kWh}$ and require 16 switchings. The examples are indicative of the need to assess the costeffectiveness of reconfiguration, which can be obtained by comparing the costs of switching and reduction in power purchase costs at loss reduction.

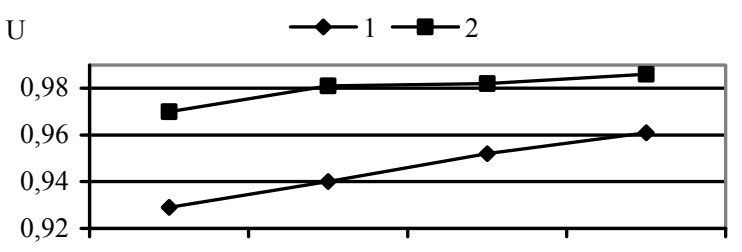

a

a1

b1

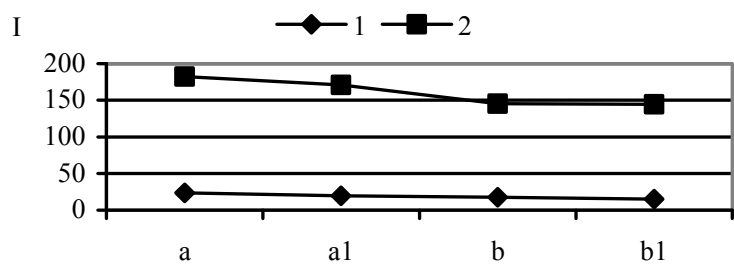

Fig. 7. Minimum 1 and average 2 voltage (U), average 1 and maximum 2 currents (I) in tie lines, a - without renewable generation and without reconfiguration, $\mathrm{b}$ - without renewable generation and with reconfiguration, a1- with renewable generation and without reconfiguration, b1- with renewable generation and with reconfiguration.

Hourly reconfiguration and involvement of renewable generation in the distribution network have a positive effect on both the reduction in losses and the decrease in the deviations in voltage and currents in the distribution network branches. Figure 6 demonstrates the plots characterizing hourly variations in voltage at nodes and currents in branches of the test network with and without reconfiguration and renewable generation. Their analysis made it possible to make the following conclusions, with their numerical confirmation demonstrated in Fig.7.

- $\quad$ Reconfiguration leads to a reduction in medium and maximum currents of feeders and fosters an increase in the number of nodes with high voltage.
- Renewable generation has a stronger effect on the reduction in average current and increase in voltage than reconfiguration. This is caused first of all by the fact that the distance of power transmission from source to load is reduced. Figure 8 shows a scheme with disconnected tie switches with power flows going from the network and renewable generation sources.

- The maximum reduction in current and increase in the minimum voltage are achieved when both renewable generation and changes in the distribution network topology by reconfiguration together affect the operation.

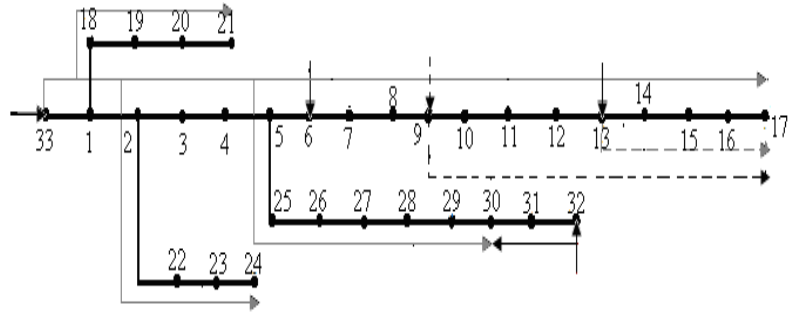

Fig. 8. Active power flows from network and renewable generation sources to the load nodes.

\section{An algorithm for optimization of daily load curves}

Optimization of daily load curves of load-controlled consumers is an additional effective way to minimize losses and reduce currents in distribution network. The problem of linear programming with constraints is applied to minimize the cost of electricity purchased at prices varying during the day

$\min \sum_{t=1}^{n_{t}} \sum_{i=1}^{n_{p}} c_{t} P_{i}^{t}$

where $n_{t}$ - the number of hourly intervals during the day; $n_{P}$ - number of load-controlled consumers; $c_{t}-$ power price at hour $t ; P_{i}^{t}-$ load of the $i$-th loadcontrolled consumer at hour t.

The constraints for each time interval include: equations of total balances of active and reactive power under constant power factor; equations of invariability of daily power consumption by each load-controlled consumer; and inequalities, determining feasible ranges of variation in power of load-controlled consumers and a slack node.

All the necessary information for solving problem (2) that lies in the determination of optimal hourly values of powers $P_{i^{*}}^{t}$ of load-controlled consumer, is determined by calculating feasible load flow [12].

The repeated calculation of a feasible load flow is made after solving problem (2) either. This is necessary for both the assessment of feasibility of all variables obtained after optimization of load curves and the assessment of an impact the optimization has on the reduction in daily energy losses. In the event that the constraints on the state variables in interval $t_{k}$ are not 
met the upper $\overline{P_{i}^{t_{k}}}$ or lower $P_{i}^{t_{k}}$ constraints on load $P_{i}^{t_{k}}$ are corrected:

$$
\begin{aligned}
& \overline{P_{i}^{t_{k}}}=P_{i}^{t_{k}}+s_{d}^{t_{k}}\left(P_{i^{*}}^{t_{k}}-P_{i}^{t_{k}}\right), \text { if } P_{i^{*}}^{t_{k}}>P_{i}^{t_{k}} ; \\
& \underline{P_{i}^{t_{k}}}=P_{i}^{t_{k}}+s_{d}^{t_{k}}\left(P_{i^{*}}^{t_{k}}-P_{i}^{t_{k}}\right), \text { if } P_{i^{*}}^{t_{k}}<P_{i}^{t_{k}},
\end{aligned}
$$

where $s_{d}^{t_{k}}>0$ - maximum feasible step of a variation in loads of load-controlled consumer in the direction of vector whose $i$-th component equals $\left(P_{i^{*}}^{t_{k}}-P_{i}^{t_{k}}\right)$.

After the limiting values in the intervals with unmet constraints are adjusted, problem (2) is solved again.

Figure 9 shows the saving costs of energy purchase at the prices varying during the day and variations in the daily load curves of load-controlled consumers at nodes 23 and 24 of a test network with the maximum total daily energy consumption equal to $10844.4 \mathrm{kWh}$.

The range of an feasible reduction in power of a load-controlled consumer from hour 8 to hour 17 is set equal to from $6 \%$ to $26 \%$.
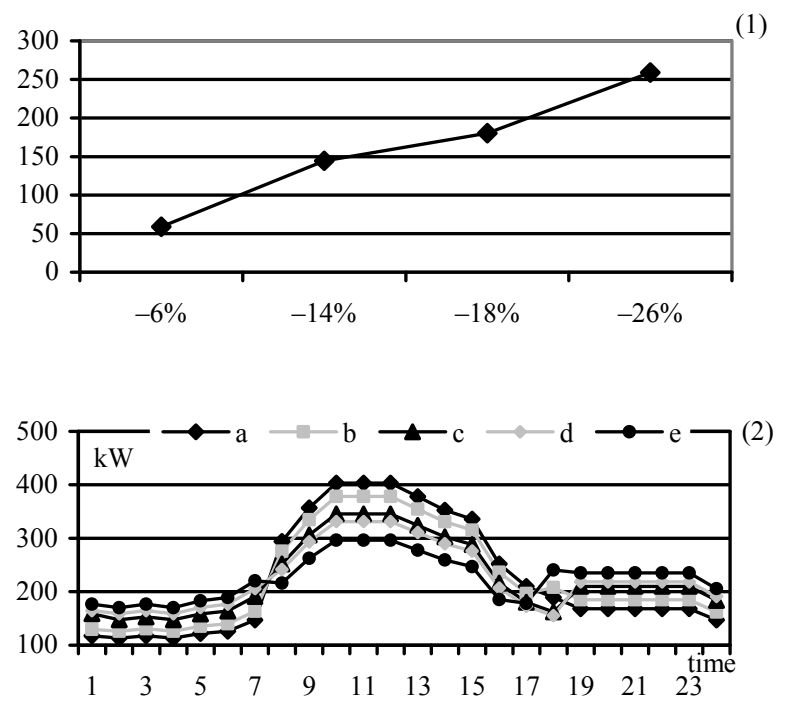

Fig. 9. A saving costs on energy (1) in conventional units and change in the daily load curves of load-controlled consumers (2) at nodes 23,24 under a feasible reduction in the maximum load at hours $8-17$ of the day by $6 \%-b, 14 \%-c), 18 \%-d$ ) and $26 \%$ - e) compared to the initial load curve a).

Figure 10 presents daily and hourly energy losses that make it possible to assess the contribution of the $26 \%$ reduction in maximum loads of the load-controlled consumer to the losses: in the distribution network without renewable generation (without reconfiguration and with reconfiguration), with renewable generation (with and without reconfiguration). Comparison of the results demonstrates that load-controlled consumers have a lesser impact on the losses than renewable generation and reconfiguration. The daily losses equal to $613.91 \mathrm{kWh}$ obtained by simultaneously using reconfiguration, renewable generation and loadcontrolled consumers are only by $23.07 \mathrm{kWh}$ lower than the losses under the joint use of renewable generation and reconfiguration. The influence of load-controlled consumers on voltage losses in distribution network was also little. The introduction of load-controlled consumers in the cases with renewable generation without reconfiguration and renewable generation with reconfiguration reduced maximum currents in the distribution network from $145.34 \mathrm{~A}$ to $134.78 \mathrm{~A}$ and from $144.275 \mathrm{~A}$ to $133.716 \mathrm{~A}$, respectively.

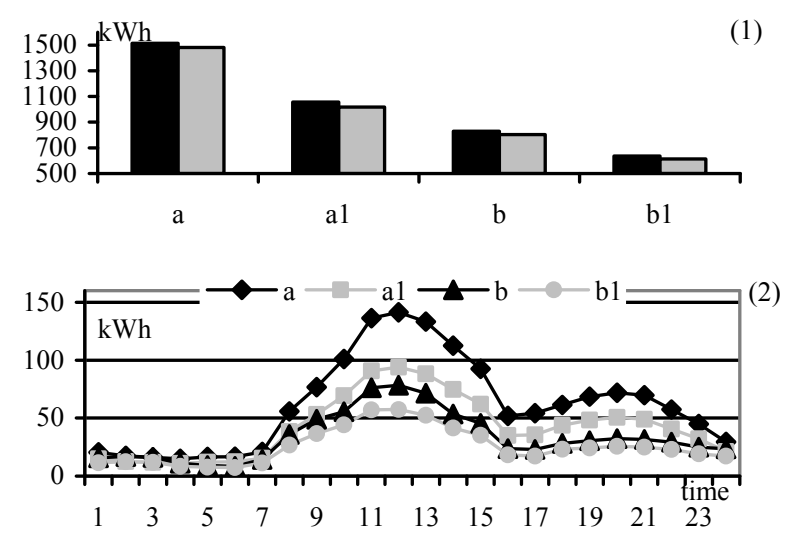

Fig. 10. Values of daily energy losses without load-controlled consumers (black) and with load-controlled consumer (grey) with a feasible reduction in their maximum power by $26 \%$ (1) and variant in hourly energy losses in the distribution network with load-controlled consumer (2) a - without renewable generation and without reconfiguration, a1 - without renewable generation and with reconfiguration, $b$ - with renewable generation and without reconfiguration, b1 - with renewable generation and with reconfiguration.

\section{Assessment of the influence of the uncertainty of load and generation data on reduction in power losses at reconfiguration of distribution network}

The mathematical mean of power losses is known to increase with a rise in the uncertainty of nodal power data [13]. The question arises if the errors of determining hourly energy losses caused by the errors in the load and generation forecasts are commensurate with the payoff from the expected decline in losses under reconfiguration of the distribution network.

To answer this question we first determined optimal switchings and hourly and daily energy losses corresponding to them for the forecast values of nodal powers for each hour of a daily load curve and renewable generation. Then, the data on hourly loads, generation and hourly configuration of the distribution network were used to calculate the probabilistic load flow, which involved the determination of the standard deviations of hourly energy losses.

The standard deviations of loads and generation of renewable generation necessary for probabilistic load flow were determined by using an inverse function of errors for a specified error of load and generation data, equal to $5 \%, 10 \%, 15 \%, 20 \%$ of their forecast values representing mathematical means. 
The inverse function of errors enables the determination of standard deviations $\sigma_{x}$ of normally distributed random variable $x$, by a specified value of interval $\Delta \varepsilon_{x}$, in which this variable will be with a set probability $p$, which in our case is equal to 0.95 ,

$$
\sigma_{x_{p=0.95}}=\Delta \varepsilon_{x} /(\sqrt{2} \operatorname{erfinv}(p))=\Delta \varepsilon_{x} / 1.96
$$

The probabilistic load flow was calculated by linear analytical method of moments. According to this method the mathematical means and covariances of nodal power at the point of solution to the nonlinear systems of steady state equations are used to calculate mathematical means and covariances of absolute values and phases of voltage, power flows and losses [14].

Interval $\Delta \varepsilon_{x}$ of a potential change in any of the indicated variables $x$, including that of energy losses, was determined by their standard deviations $\Delta \varepsilon_{x}=\sqrt{2} \operatorname{erfinv}(p) \sigma_{x}$. An interval of a potential variation in the random value as a percentage of mathematical mean $\mu_{x}$ was determined as $\Delta \varepsilon_{x} \%=\sqrt{2} \operatorname{erfinv}(p) \sigma_{x} \cdot 100 \% / \mu_{x}$.
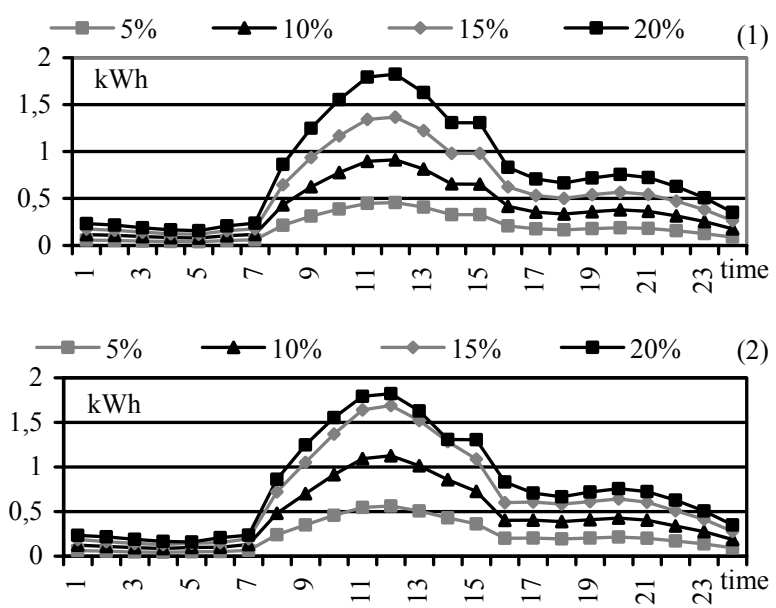

Fig. 11. Standard deviation of hourly energy losses for the scheme with renewable generation and with reconfiguration (1), and without renewable generation and with reconfiguration (2).

Figure 11 demonstrates standard deviations of hourly energy losses for distribution network with reconfiguration (with and without renewable generation). The deviations show that the hourly deviations of energy losses in both cases do not exceed $2 \mathrm{kWh}$. At the same time the hourly errors of determining energy losses, i.e. intervals of deviation of hourly energy losses from their mathematical means (Fig.12), for a $20 \%$ error of the nodal power forecast do not exceed $8 \%$.

The standard deviations and errors of daily energy losses presented in Fig.13 show that at the maximum error in the forecast they do not exceed $6 \mathrm{kWh}$ and $1.5 \%$ of the mathematical mean of losses.

The presented results confirm that with an increase in uncertainty of data on nodal power, hourly and daily energy losses rise, which cannot serve as grounds for the rejection of the reconfiguration aimed at reducing losses in the network.

$\% \rightarrow-5 \% \longrightarrow 10 \% \multimap 15 \% \rightarrow-20 \%$
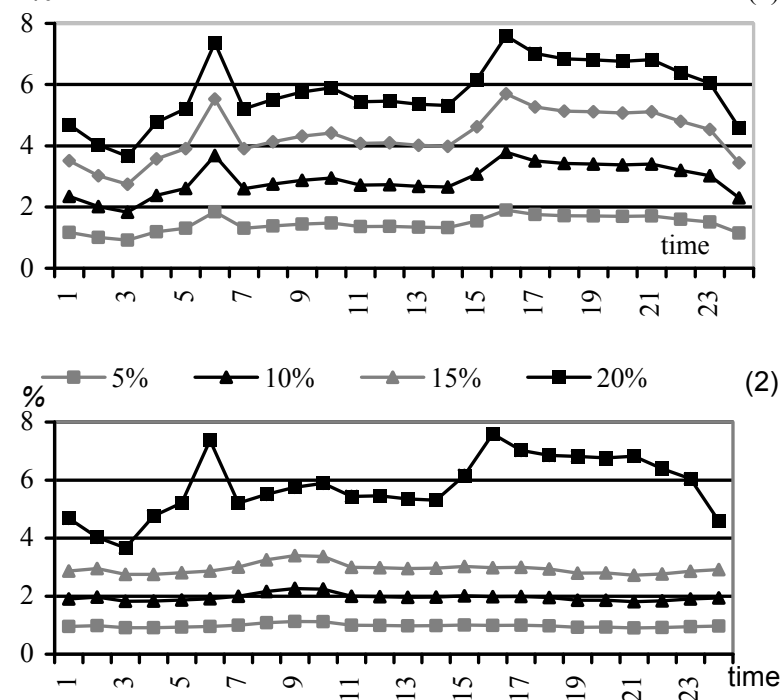

Fig. 12. Errors in the determination of hourly energy losses for the scheme with renewable generation and with reconfiguration (1), and without renewable generation and with reconfiguration (2).

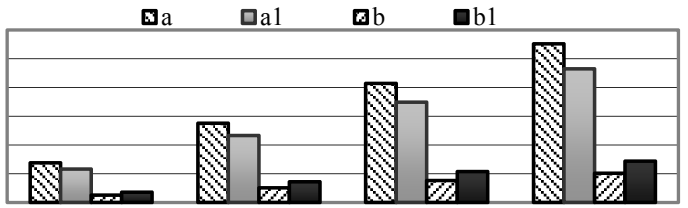

Fig. 13. Standard deviations (a, a1) (kWh) and errors (b, b1) (\%) of daily energy losses for the network with renewable generation and with reconfiguration $(a, b)$, without renewable generation and with reconfiguration $(\mathrm{a} 1, \mathrm{~b} 1)$.

\section{Conclusions}

1. A topological algorithm for distribution network reconfiguration is generated to reduce power losses, and the possibility of its application to the hourly reconfiguration of the network, in particular with available renewable energy sources in the distribution network is shown.

2. An algorithm is suggested to optimize daily load curve of a load-controlled consumer by the criterion of minimization of energy purchase costs, which also makes it possible to reduce the losses in distribution network.

3. The paper is focused on the study of the influence of renewable energy sources, hourly reconfiguration, optimization of load curve of load-controlled consumers, and their joint use on the daily energy losses.

4. The possibility of choosing an invariable distribution network configuration which provides the daily energy losses comparable with the losses determined at the hourly reconfiguration of distribution network is demonstrated. 
5. The numerical results confirmed the effectiveness of the suggested algorithms.

6. The study shows that the errors in the calculation of losses rise with an increase in the errors of the nodal power forecast, but they do not have a decisive influence on the reduction in losses at reconfiguration.

\section{References}

1. O.N. Voitov, I.I. Golub, L.V. Semenova, Elektrichestvo 9, 38 (2010)

2. M. Emmanuel, R. Rayudu, JNCA 74, 133 (2016)

3. M.R. Dorostkar-Ghamsari, M. Fotuhi-Firuzabad, M. Lehtonen, A. Safdarian, IEEE Trans. on Power Systems 31, 1879 (2016)

4. H. Ahmadi, J.R. Marti, SEGAN 1, 1 (2015)

5. D. Zhang, Z. Zhang, I. Fu, Electr. Power Sys. Res. 77, 10 (2007)
7. N.A. Melnikov The matrix method for analysis of electric circuits, M: Energiya. 288 (1972) (in Russian)

8. M. Behrangrad, Renew. Sustain. Energy Rev. 47, 270 (2015)

9. C.L. Su. IEEE Trans. Power Syst. 25, 786 (2010)

10. A.A. Glazunov, A.A Glazunov. Electric networks and systems (Gosenergoizdat, Moskow, 1960)

11. M.E Baran, F.F. Wu, IEEE Trans. Power Del. 4, 1401 (1989)

12. N.A. Murashko, Y.A. Okhorzin, L.A. Krumm Analysis and control of steady states of electric power systems (Nauka, Novosibirsk, 1987)

13. V.Z. Manusov, A.V. Mogilenko, Elektrichestvo, 3, 2 (2003)

14. E.V Boloev, O.N. Voitov, I.I. Golub, Akta Energetica 2(23), 91 (2015) 